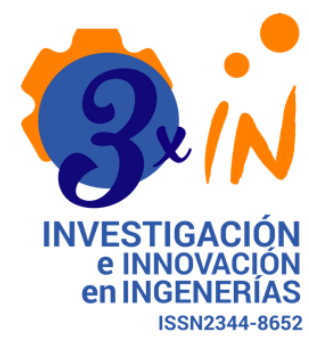

Open Access

Recibido:

2 de abril de 2020

Aceptado:

3 de agosto de 2020

Publicado:

30 de septiembre de 2020

Correspondencia:

hugoordonez@unicauca.edu.co

DOI:

https://doi.org/10.17081/invinno.8.2 .3892

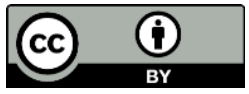

(C) Copyright: Investigación e Innovación en Ingenierías

\section{Modelado y simulación de una marcha bípeda usando optimización paramétrica}

\section{Modeling and simulating biped movement through parameter- based optimization}

\author{
Cristian Méndez Rodríguez (iD), Armando Ordoñez (iD), Cristian Camilo Ordoñez \\ Fundación Universitaria de Popayán, Colombia \\ Hugo Ordoñez Erazo \\ Universidad del Cauca, Colombia
}

Resumen

Objetivo: Modelar dinámica y geométricamente una estructura bípeda de 6 grados de libertad (GDL), dado que uno de los sistemas más complicados de modelar es la biomecánica de la marcha humana. Metodología: Se aplicó la técnica de optimización paramétrica para calcular las trayectorias que definen una marcha humana cíclica. Los parámetros geométricos y dinámicos del bípedo fueron tomados del robot Hydroid, que está basado en el modelo morfológico propuesto por HANAVAN, caracterizando fielmente el cuerpo humano. Resultados: Los modelos matemáticos propuestos, permitieron calcular las posiciones, velocidades, y aceleraciones angulares que adopta cada articulación del bípedo en diferentes instantes de tiempo para realizar un movimiento cíclico. Además, fueron encontrados los torques articulares óptimos (criterio de mínimo consumo energético) requeridos para realizar un movimiento específico: marcha compuesta de fases consecutivas de simple apoyo e impacto, sin considerar la fase de rotación del pie. Con los datos calculados, fue realizada una simulación. Conclusiones: Finalmente, es posible indicar que el uso de herramientas como la optimización paramétrica, y el modelado matemático, permite grandes desarrollos en áreas como la mecatrónica, y la biomédica, donde son requeridos patrones de marcha para ser aplicados en sistemas robóticos, dispositivos de rehabilitación, e identificación de patologías.

Palabras claves: Marcha bípeda, modelo dinámico, optimización paramétrica, trayectorias articulares, criterio energético.

\begin{abstract}
Objective: This work dynamically and geometrically models a biped structure with 6 degrees of freedom (DOF) because one of the most difficult systems to model is the biomechanics of human walking. Methodology: This study used the parameter-based optimization technique to calculate the cyclica trajectories that define human walking. The geometric and dynamic biped parameters were adopted from the Hydroid robot as it is based on the morphological model proposed by HANAVAN that faithfully characterizes the human body. Results: Using the proposed mathematical models, the positions, speeds, and angular accelerations that each biped joint adopts at different moments of time when performing cyclical movements are estimated. In addition, the study identifies the optimal joint torques (criterion of minimum energy consumption) required to perform a specific movement: walking composed of consecutive simple support and impact phases, disregarding the foot rotation phase. Then, a simulation is performed based on these data. Conclusions: According to the results reported by the study, parameterbased optimization and mathematical modeling will result in extensive development in the mechatronics and biomedical fields, wherein movement patterns are required for their application in robot systems and for rehabilitation and pathology identification devices.
\end{abstract}

Keywords: Biped movement, dynamic model, parameter-based optimization, joint trajectories, energy criteria.

Como citar (IEEE): C. Méndez - Rodríguez, A. Ordoñez, C.C. Ordoñez, H. Ordoñez - Erazo, "Modelado y simulación de una marcha bípeda usando optimización paramétrica", Investigación e Innovación en Ingenierías, vol. 8, nº 2, 2020. DOI: https://doi.org/10.17081/invinno.8.2.3892 


\section{Introducción}

Durante décadas se han realizado extensos estudios sobre la marcha bípeda en diferentes áreas del conocimiento. Desde una perspectiva biológica para explicar el origen del hombre, la medicina para analizar patologías, y para la rehabilitación de pacientes asistidos por robots, y desde la ingeniería en el desarrollo de sistemas bípedos robóticos [1, 2, 3, 4, 5, 6, 7]. Estas investigaciones han dado origen a campos de investigación como la evolución de la marcha, minimización del consumo energético, generación de patrones de marcha, y control de estabilidad [8]. En la actualidad, un reto que se presenta es generar estos movimientos de marcha bípeda (fieles al movimiento humano), integrando los múltiples campos de investigación, mediante el desarrollo de algoritmos computacionales.

En el estudio biomecánico de la marcha humana, es posible considerar el desarrollo de diferentes métodos que permiten generar un ciclo de marcha o también llamados "generadores de movimiento" [9, 10, 11]. En los estudios $[12,13]$, se destacan tres tipos de marcha que caracterizan un movimiento antropomórfico cíclico:

- Sucesión de fases de simple apoyo separados por impactos impulsivos, denominada "marcha en fase de simple suporte (SS) pie plano más impacto pie plano",

- Una fase de doble apoyo con rotación de los dos pies,

- Una fase de rotación del pie de apoyo alrededor del borde frontal en el momento de fase de simple apoyo, denominada "punta-talón, punta-talón".

Para el desarrollo de este estudio, es adoptado el primer tipo de marcha, debido a que este ofrece mayores garantías a la estabilidad de la estructura bípeda, además de ser el tipo de movimiento que menos gasto energético demanda (menor valor en torques articulares) [13]. Para la generación de este patrón de marcha, es usado el método de optimización paramétrica, el cual permite calcular las posiciones, velocidades, y aceleraciones articulares óptimas. El problema de optimización es planteado teniendo en cuenta: i) las variables a optimizar: son estimadas con base en el modelo dinámico, geométrico, y de impacto de la estructura bípeda; ii) el criterio de optimización: el objetivo es minimizar el consumo energético; iii) restricciones de optimización: restricciones no lineales que garantizan la ejecución óptima de la marcha.

Un primer objetivo de este estudio es realizar el cálculo simbólico de los modelos dinámico, geométrico, y de impacto de la estructura bípeda en 2 dimensiones. Con base en estos modelos, es propuesto un segundo objetivo, que corresponde a plantear y resolver un problema de optimización paramétrico. Lo anterior nos permite calcular las trayectorias articulares óptimas (mínimo consumo energético), para que la estructura bípeda realice el movimiento de marcha cíclico definido: Simple Soporte (SS) Pie Plano más Impacto Pie Plano. 
Este artículo se estructura de la siguiente forma: la sección 2 describe el método de investigación empleado, el cual consiste en primera instancia en presentar los parámetros geométricos y dinámicos de la estructura bípeda; en segundo momento, en plantear el modelo dinámico y de impacto de la estructura. Finalmente, en la sección 3 es propuesto y resuelto el problema de optimización paramétrico que llevan a la generación de una marcha óptima, presentando los resultados obtenidos, las trayectorias articulares y torques articulares óptimos para realizar el tipo de marcha establecida. La sección 4 expone las conclusiones.

\section{Metodología}

\section{Presentación de la estructura bípeda}

El modelo 2D de la estructura bípeda es definida a partir de las características del robot Hydroïd [14]. Este robot está diseñado con base en parámetros geométricos y dinámicos similares al modelo propuesto por Hanavan que caracteriza fielmente la biomecánica del ser humano [15]. Hydroïd está compuesto de un tronco y de dos piernas idénticas, cada pierna tiene tres articulaciones (tobillo, rodilla y cadera) (Figura 1). La estructura cinemática arborescente tiene 6 grados de libertad (GDL), y los parámetros geométricos y dinámicos de la estructura son presentados en la Tabla 1.

Figura 1. Estructura cinemática arborescente de la estructura bípeda 2D

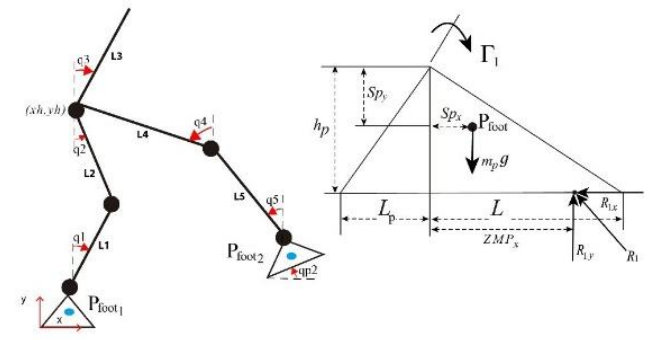

Fuente: Elaboración propia

Tabla 1. Parámetros físicos de la estructura bípeda

\begin{tabular}{|c|c|c|c|c|c|}
\hline Mecanismo & Descripción & $\begin{array}{l}\text { Masa } \\
\mathrm{Kg}\end{array}$ & $\begin{array}{l}\text { Longitud } \\
m\end{array}$ & $\begin{array}{l}\text { Centro de Masa } \\
m\end{array}$ & $\begin{array}{l}\text { Inercia } \\
\mathrm{Kg} \cdot \mathrm{m}^{2}\end{array}$ \\
\hline \multirow[t]{4}{*}{ Humano } & Pie (Cuerpos Pie1, Pie2) & 0.678 & $\begin{array}{c}\mathrm{L}_{\mathrm{p}}=0.072 \\
\mathrm{~L}_{\mathrm{d}}=0.135 \\
\mathrm{~h}_{\mathrm{p}}=0.06425\end{array}$ & $\begin{array}{l}s p_{x}=0.0135 \\
s p_{y}=0.03212\end{array}$ & 0.001753 \\
\hline & Tibia (cuerpo. 1 y 5) & 2.188 & 0.392 & 0.16856 & 0.02765 \\
\hline & Muslo (cuerpo. 2 y 4) & 5.025 & 0.392 & 0.16856 & 0.06645 \\
\hline & Tronco (cuerpo 3) & 29.27 & 0.5428 & 0.192065 & 0.8149635 \\
\hline
\end{tabular}

Fuente: Elaboración propia 
Las coordenadas generalizadas del bípedo son ángulos absolutos, definidos con respecto al eje vertical, paralelo al vector de gravedad, así, el espacio articular del mecanismo está dado por:

$$
\mathrm{q}=[\mathrm{qp} 1, \mathrm{q} 1, \mathrm{q} 2, \mathrm{q} 3, \mathrm{q} 4, \mathrm{q} 5, \mathrm{qp} 2]
$$

Dónde $q p 1=0$, ya que el pie uno está en soporte plano con el suelo. De esta manera, la generación de la trayectoria cíclica (todos los pasos idénticos) modelada en este trabajo, está compuesta de fases de simple apoyo pie plano y de impacto pie plano, es decir, consiste en una sucesión de fases de simple apoyo separado por impactos impulsivos o instantáneos (Figura 2).

Figura 2. Movimiento: simple soporte pie plano + impacto pie plano (Incluyendo impactos impulsivos)

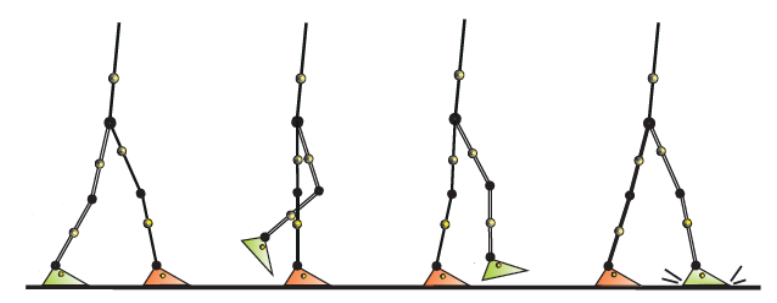

Fuente: [16]

\section{Modelado de la estructura bípeda}

Con base en el formalismo de Lagrange y en la técnica presentada en [17], el modelo dinámico en fase de simple apoyo puede ser escrito como se muestra en (2). Esta expresión es válida solamente si el pie de apoyo permanece fijo en la conexión con el suelo, es decir, se deben cumplir tres condiciones: i) no exista deslizamiento del pie en soporte, ii) no debe haber rotación del pie de apoyo, y iii) no haya despegue o desprendimiento del pie de apoyo con el suelo.

$A(q) \ddot{q}+C(q, \dot{q}) \dot{q}+G(q)=B \Gamma$

Dónde:

q es el vector de coordenadas generalizadas, $\dot{\mathrm{q}}$ y $\ddot{\mathrm{q}}$ la primera y segunda derivadas de $q$ con respecto al tiempo,

- $\Gamma=\left[\begin{array}{llll}\Gamma_{1} & \Gamma_{1} & \ldots & \Gamma_{n}\end{array}\right]^{\mathrm{T}}$ es el vector de torques articulares,

- $\mathrm{A}(\mathrm{q}) \in \mathbb{R}^{\mathrm{nxn}}$ es la matriz de inercia, que debe ser simétrica y definida positiva,

- $\mathrm{C}(\mathrm{q}, \dot{\mathrm{q}}) \in \mathbb{R}^{\mathrm{nxn}}$ representa las fuerzas de Coriolis y las fuerzas

- Centrifugas, 
- $\mathrm{G}(\mathrm{q}) \in \mathbb{R}^{\mathrm{n} \times 1}$ denota el vector de efectos de la gravedad,

- $B \in \mathbb{R}^{\text {nxn }}$ es la matriz de accionamiento, es definida constante e invertible,

- $\mathrm{n}=6$, el número de articulaciones que tiene la estructura bípeda.

La fuerza de reacción del suelo sobre el pie que está en soporte, es llamada fuerza $\mathrm{R}_{1}$ (Figura 1), durante esta fase de simple apoyo, es calculada mediante el equilibrio dinámico de la estructura bípeda, que puede ser escrita:

$\left[\begin{array}{l}\mathrm{R}_{1 \mathrm{x}} \\ \mathrm{R}_{1 \mathrm{y}}\end{array}\right]=\mathrm{m}\left[\begin{array}{l}\ddot{\mathrm{X}}_{\mathrm{g}} \\ \ddot{\mathrm{Y}}_{\mathrm{g}}\end{array}\right]+\mathrm{m}\left[\begin{array}{l}0 \\ \mathrm{~g}\end{array}\right]$

$\mathrm{m}$ es la masa total del bípedo; $\mathrm{x}_{\mathrm{g}} \mathrm{y} \mathrm{y}_{\mathrm{g}}$ son las componentes horizontal $\mathrm{y}$ vertical del centro de gravedad ( $\mathrm{CdG}$ ) del bípedo; $y \mathrm{R}_{1 \mathrm{x}}$ y $\mathrm{R}_{1 \mathrm{y}}$ son las componentes horizontal y vertical de la fuerza de reacción del suelo sobre el pie que permanece en soporte.

Además, es importante considerar el concepto de ZMP (Zero-Moment Point), el cual ayuda a garantizar la estabilidad en la marcha del bípedo. En varios trabajos se profundiza sobre este concepto $[9,18,19]$. El ZMP debe estar contenido en la zona de apoyo, con el fin de asegurar que el pie permanezca en soporte plano con el suelo, es decir, para garantizar que el pie no se deslice, ni gire o rote alrededor de una de sus aristas (ver Figura 1). El ZMP puede ser calculado a partir del equilibrio de los momentos de torsión alrededor del pie de apoyo (4):

$\mathrm{ZMP}_{\mathrm{x}}=\frac{\Gamma_{1}+\mathrm{Sp}_{\mathrm{x}}+\mathrm{m}_{\mathrm{p}} \mathrm{g}-\mathrm{h}_{\mathrm{pR}} \mathrm{R}_{1 \mathrm{x}}}{\mathrm{R}_{1 \mathrm{y}}}$

- $\mathrm{h}_{\mathrm{p}}$ es la altura del pie,

- $\mathrm{Sp}_{\mathrm{x}}$ es la posición del centro de masa del pie con respecto al tobillo,

- $\mathrm{m}_{\mathrm{p}}$ es la masa del pie,

- y $\Gamma_{1}$ el torque ejercido por la articulación del tobillo. 


\section{Modelo de impacto}

En la marcha bípeda, el impacto se produce al final de la fase de simple apoyo, cuando la pierna que está desplazándose o en balanceo toca el suelo. Con respecto a esto, dos casos pueden presentarse:

Caso 1: el pie que esta inicialmente en apoyo despega del suelo luego del impacto.

Caso 2: los dos pies permanecen en contacto con el suelo.

Para el primer caso, tres situaciones se deben cumplir:

- La magnitud de la componente vertical de la velocidad de cada arista del pie que despega del suelo justo luego del impacto, debe ser positiva.

- La fuerza de reacción del suelo sobre la pierna que despega del suelo debe ser nula, es decir: $\mathrm{R}_{1}=0$.

- Luego del impacto, se considera que la magnitud de la componente vertical de la fuerza de reacción del suelo sobre el pie que impacta (nuevo pie en apoyo), debe ser positiva [20].

Para el cálculo del modelo de impacto, es necesario considerar el modelo dinámico en fase de doble apoyo. Para esto, las posiciones o conFiguraciones articulares de la estructura serán definidas por el vector $\mathrm{X}=$ $\left[\begin{array}{llllllll}\mathrm{q}_{1} & \mathrm{q}_{2} & \mathrm{q}_{3} & \mathrm{q}_{4} & \mathrm{q}_{5} & \mathrm{qp}_{1} & \mathrm{qp}_{2} \mathrm{xh} & \mathrm{yh}\end{array}\right]^{\mathrm{T}}$, dónde $\mathrm{xh}$ y yh son las coordenadas cartesianas que indican la posición de la cadera, y $\mathrm{qp}_{1}=0$ ya que el pie en soporte está en contacto plano con el suelo. El modelo dinámico en fase de doble apoyo del bípedo puede ser escrito así:

$A(X) \ddot{X}+C(X, \dot{X}) \dot{X}+G(X)=B \Gamma+J_{1}^{T} R_{1}+J_{2}^{T} R_{2}$

- $\mathrm{R}_{1} \mathrm{y} \mathrm{R}_{2} \in \mathbb{R}^{3 \times 1}$ son los vectores de reacción entre el suelo y los dos pies,

- $\mathrm{J}_{1} \mathrm{y} \mathrm{J}_{2}$ son las matrices Jacobianas para los dos pies.

Considerando el Caso 1, se supone la fase de doble apoyo instantánea, el modelo de impacto es obtenido integrando la ecuación (5) durante la duración del impacto (tiende a cero, porque es un impacto instantáneo). El modelo de impacto es:

$\left[\begin{array}{c}\cdot \dot{\mathrm{X}}^{+} \\ \mathrm{R}_{2}\end{array}\right]=\left[\begin{array}{cc}\mathrm{A} & -\mathrm{J}_{2}^{\mathrm{T}} \\ \mathrm{J}_{2} & 0_{3 \times 3}\end{array}\right]^{-1}\left[\begin{array}{c}\mathrm{A} \cdot \dot{\mathrm{X}}^{-} \\ 0_{3 \times 3}\end{array}\right]$

$\mathrm{X}^{-}$(X punto menos) son las velocidades articulares del bípedo justo antes del 
impacto, y X+ (X punto más) las velocidades justo después del impacto. Además, la velocidad lineal del pie que llega al suelo es nula, es decir: $\mathrm{J}_{2} \mathrm{X}^{+}=0$. $R_{2} \in \mathbb{R}^{3 \times 1}$, y representa las reacciones impulsivas del suelo sobre el pie de apoyo (al momento del impacto). De este modelo es posible obtener el vector de velocidades articulares luego del impacto y las fuerzas impulsivas.

Además, es necesario considerar las siguientes condiciones:

- El impacto existe al instante preciso donde el pie en balanceo choca contra el suelo y genera un contacto pie plano.

- El impacto es instantáneo (impulsivo).

- La conFiguración del bípedo es constante durante el impacto (cuerpos indeformables).

\section{Generación de una trayectoria óptima}

Es usado un proceso de optimización paramétrica [7, 10, 21, 22] para definir una marcha óptima (cíclica): marcha en fase de simple apoyo pie plano, más impacto pie plano. Para definir la evolución de las variables articulares en el ciclo de marcha son empleadas funciones splines cubicas. Para este caso, son fijadas las posiciones y las velocidades articulares al inicio de marcha $\left(q_{\text {ini }} \mathrm{y} \dot{\mathrm{q}}_{\text {ini }}\right)$ (12 parámetros), las posiciones y velocidades articulares al final del paso $\left(\mathrm{q}_{\text {fin }} \mathrm{y} \dot{\mathrm{q}}_{\text {fin }}\right)$ (12 parámetros), así como las posiciones articulares en la mitad del paso $\left(\mathrm{q}_{\text {int }}\right)$ (6 parámetros). Estas últimas posiciones nos permitirán evitar algún tropiezo del pie que está en balanceo contra el suelo. De esta manera, es necesario tener 30 parámetros que ayuden a definir el movimiento óptimo.

Este tipo de problemas de optimización paramétrica pueden ser resueltos con ayuda de un algoritmo de tipo SQP (Sequential Quadratic Programming) [23], donde es necesario determinar: las variables, el criterio, y las restricciones.

\section{Variables de optimización}

Debido a la condición cíclica del movimiento, es posible realizar los siguientes cálculos:

- Las posiciones articulares iniciales $\left(\mathrm{q}_{\mathrm{ini}}\right)$ y las velocidades articulares iniciales $\left(\dot{\mathrm{q}}_{\text {ini }}\right)$ pueden ser calculadas a partir de las posiciones y velocidades articulares luego del impacto $\mathrm{q}^{+} \mathrm{y} \dot{\mathrm{q}}^{+}$(ecuación 6). 
- Considerando la fase de doble soporte instantáneo, con un impacto de pie plano al final del paso, son calculadas las variables articulares $\mathrm{q}_{1}, \mathrm{q}_{2}, \mathrm{q}_{4}, \mathrm{q}_{5}$ usando el modelo geométrico inverso de la estructura. Para realizar este cálculo, se debe conocer la posición cartesiana de la cadera al final del paso (xh, yh), la longitud del paso (d), y la orientación del tronco al final del paso $\left(\mathrm{q}_{3}(\mathrm{~T})\right)$; donde $\mathrm{T}$ es la duración del paso, $\mathrm{T}=\mathrm{d} / \mathrm{V}$ ( $\mathrm{d}$ es la distancia del recorrido del paso y $\mathrm{V}$ es la velocidad de marcha, que es fijada a $0.5 \mathrm{~m} / \mathrm{s}$ ). Lo anterior, permite encontrar las posiciones articulares al final del paso $\left(\mathrm{q}_{\mathrm{fin}}\right)$.

Los 30 parámetros necesarios para definir la trayectoria de marcha pueden ser reducidos a 16 variables de optimización, las cuales son:

- 4 parámetros: posiciones cartesianas de la cadera al final del paso (xh, yh), la longitud del paso (d), y la orientación del tronco al final del paso $\left(\mathrm{q}_{3}(\mathrm{~T})\right)$.

- 6 parámetros: velocidades articulares al final del paso $\left(\dot{\mathrm{q}}_{\mathrm{fin}}\right)$.

- 6 parámetros: las posiciones intermedias $\left(\mathrm{q}_{\mathrm{int}}\right)$.

\section{Criterio de optimización}

El objetivo es encontrar una trayectoria óptima que minimice el criterio energético $\mathrm{C}_{\Gamma}$ (normalización de los torques por la unidad de distancia recorrida d durante un paso de marcha cíclico).

$\mathrm{C}_{\Gamma}=\frac{1}{\mathrm{~d}} \int_{0}^{\mathrm{T}} \Gamma(t)^{T} \Gamma(t) d t$

Dónde:

- d corresponde a la longitud del paso,

- T a la duración del paso,

- $\quad \Gamma$ es el conjunto de torques articulares del bípedo.

\section{Las restricciones de optimización}

Con el objetivo de que la trayectoria de marcha calculada sea realizable, es necesario tener en cuenta dos tipos de restricciones:

i. Restricciones de contacto con el suelo durante la fase de simple apoyo e impacto pie plano (ecuación 8): 
- Restricción de no desprendimiento del pie que está en soporte: la fuerza de reacción del suelo sobre el pie que está en soporte debe ser siempre positiva [12].

- Restricción de no deslizamiento del pie que está en soporte.

- Restricción de no rotación del pie que está en soporte: el pie en apoyo debe estar en contacto plano con el suelo (toda su superficie en contacto). Para esto, se define una restricción sobre la posición del ZMP.

- Restricción velocidades lineales del pie en balanceo: para validar el modelo de impacto, es necesario verificar que las velocidades lineales del pie (punta, talón) que está en balanceo deben ser siempre positivas.

$$
\begin{gathered}
\mathrm{R}_{1 \mathrm{y}}>0 \\
\mu \mathrm{R}_{1 \mathrm{y}} \geq\left|\mathrm{R}_{1 \mathrm{x}}\right| \\
-L_{\mathrm{p}} \leq \mathrm{ZMP}_{\mathrm{x}} \leq L \\
\left\{\begin{array}{c}
V_{\text {tiptoe }} \geq 0 \\
V_{\text {heel }} \geq 0
\end{array}\right.
\end{gathered}
$$

Restricciones Tecnológicas: son limitaciones introducidas sobre los torques, las posiciones y velocidades articulares. En la ecuación 9 se describen estas restricciones:

$$
\begin{aligned}
\left|\Gamma_{i}\right|-\Gamma_{i, \max } \leq 0 \\
\left|\dot{\mathrm{q}}_{\mathrm{i}}\right|-\dot{\mathrm{q}}_{\mathrm{i} \text { max }} \leq 0 \quad \text { para } \mathrm{i}=1, \ldots, \mathrm{n} \\
\mathrm{q}_{\mathrm{i}, \text { min }} \leq \mathrm{q}_{\mathrm{i}} \leq \mathrm{q}_{\mathrm{i}, \text { max }}
\end{aligned}
$$

\section{Resultados}

Resolviendo el problema de optimización propuesto, es calculada la trayectoria de marcha óptima del bípedo, la cual está definida por las posiciones, velocidades y aceleraciones articulares. La velocidad de marcha establecida es de $0.5 \mathrm{~m} / \mathrm{s}$ (velocidad promedio de un humano). La Figura 3 presenta la evolución de las posiciones articulares del bípedo en diferentes instantes de tiempo para dar un paso. Debido a que se trata de una marcha cíclica, todos los pasos serán idénticos. El valor de la posición articular del tobillo (pie en apoyo) es igual a cero, ya que el pie siempre está en soporte plano con el suelo. 
Figura 3. Evolución en el tiempo de las posiciones articulares del bípedo, para una velocidad de marcha de $0.5 \mathrm{~m} / \mathrm{s}$

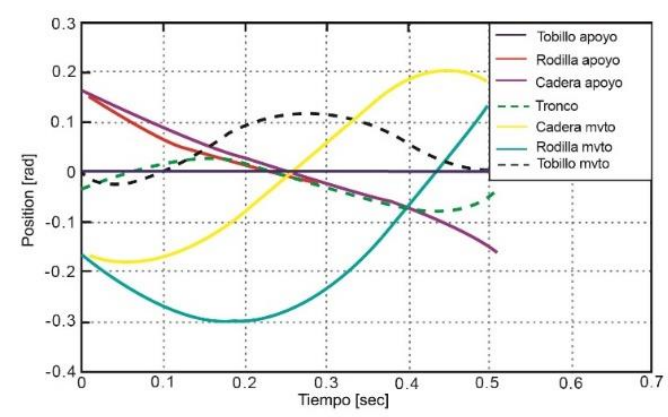

Fuente: Elaboración propia

Para encontrar el valor de los torques articulares que permitan llevar a cabo la trayectoria de marcha, se despeja la variable $\Gamma$ de la ecuación (2), obteniendo:

$\Gamma=(B)^{-1} A(q) \ddot{q}+C(q, \dot{q}) \dot{q}+G(q)$

Los valores calculados para los torques articulares son los valores óptimos, es decir los que menos gasto energético requieren para cumplir con las condiciones de la trayectoria definida. En la Figura 4 son presentadas las curvas de los torques articulares necesarios para que el bípedo de un paso.

La Figura 5 muestra la evolución de la altura del pie en balanceo, esto nos ayuda a verificar que las posiciones cartesianas de la punta y el talón del pie que está en balanceo sean positivas (eje y), para garantizar que no suceda ningún tropiezo que afecte la estabilidad del bípedo. De igual manera, de esta Figura, es posible comprobar que el impacto está cumpliendo con la condición establecida: contacto pie plano; es decir, que toda la superficie del pie impacte plenamente el suelo. Lo anterior se ve claramente, cuando las dos curvas llegan a una altura cero al mismo momento, indicando que el impacto de la punta y el talón del pie se producen en el mismo instante. 
Figura 4. Evolución en el tiempo de los torques articulares óptimos, para una velocidad de marcha de $0.5 \mathrm{~m} / \mathrm{s}$

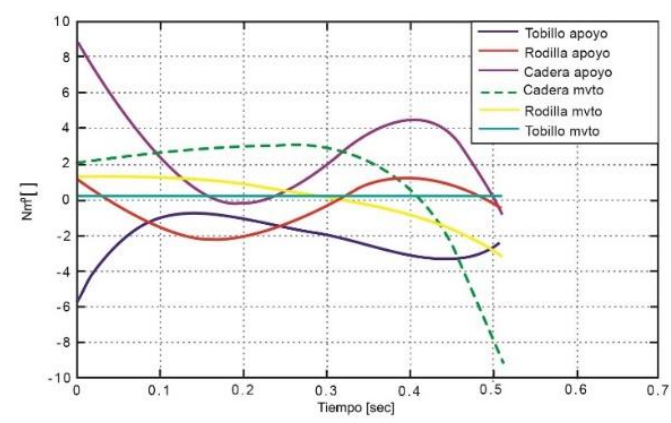

Fuente: Elaboración propia

Figura 5. Evolución de la altura del pie que está en balanceo durante un paso

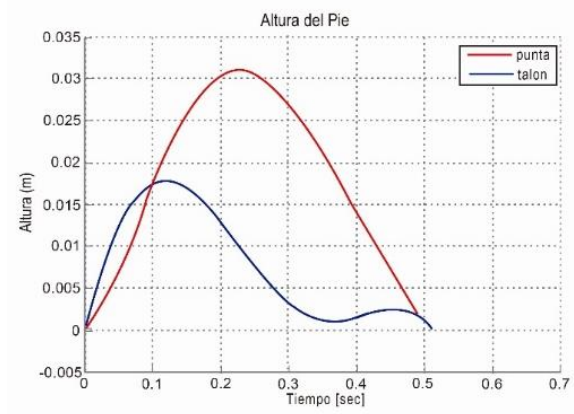

Fuente: Elaboración propia

Finalmente, fue realizada una simulación del bípedo realizando la marcha óptima programada (ver Figura 6).

Figura 6. Simulación del bípedo realizando la marcha programada

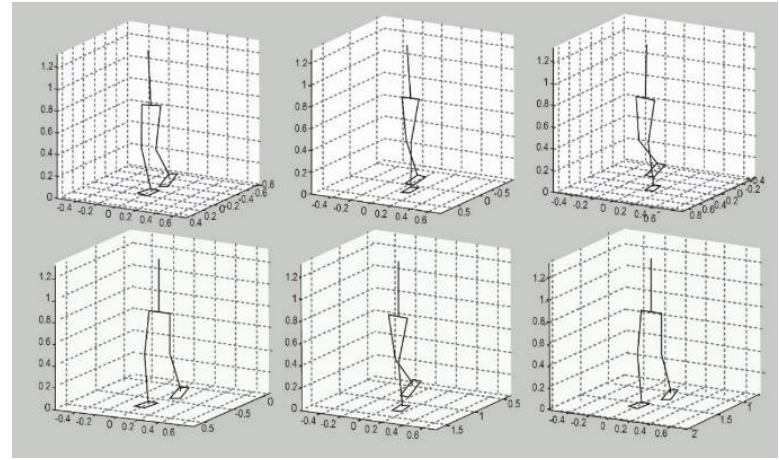

Fuente: Elaboración propia 


\section{Conclusiones}

Usando las características geométricas y dinámicas del robot Hydroïd, fue propuesto un problema de optimización paramétrica para obtener una marcha cíclica: simple soporte pie plano más impacto pie plano. Para solucionar el problema se tuvieron en cuenta los diferentes modelos dinámicos, geométricos, y de impacto relacionados con la estructura bípeda. La trayectoria de marcha es planteada considerando algunas restricciones no lineales que garantizan la estabilidad del bípedo, como por ejemplo: no despegue del pie en movimiento, no deslizamiento, y no rotación. Además, el criterio optimizado es el consumo energético, con el objetivo de garantizar el menor gasto de energía en la trayectoria realizada.

Obtenida la trayectoria mediante el método de optimización paramétrica, se verifico que la marcha bípeda calculada cumpliera con las atributos y restricciones planteados. Se realizaron simulaciones de la estructura bípeda realizando el movimiento encontrado.

Este tipo de estudios son importantes en áreas como la física médica, donde son requeridos generadores de marcha para ser aplicados a sistemas de rehabilitación, dispositivos robóticos, análisis de patologías relacionadas con la marcha humana, etc. La perspectiva es extender los resultados obtenidos para un modelo bípedo 3D, y además usar estos datos para realizar el modelado y simulación de una persona usando un sistema de rehabilitación (exoesqueleto).

\section{Referencias bibliográficas}

1. P. Medina and E. Mancilla, "Evolución de la locomoción bípeda humana: el nivel socioeconómico como factor ambiental", Antropo, vol. 32, pp. 15-24, 2015.

2. C. O. Lovejoy, "The Origin of Man", Science, vol. 211, n. 4480, pp. 341-350, $2009 . \quad$ DOI: https://doi.org/10.1126/science.211.4480.341

3. B. Wood, "Origin and evolution of the genus Homo", Nature, vol. 355, pp. 783-790, $1992 . \quad$ DOI: https://doi.org/10.1038/355783a0

4. T. Sasaki, A. Yozu, S. Tokushige, Y. Ugawa, S. Tsuji, and M. Hamada, "A quantitative analysis of bipedal and quadrupedal 
gait in Parkinson's disease", J. Neurol. Sci., vol. 381, p. 730, 2017. DOI: https://doi.org/10.1016/j.jns.2017.08.2057

5. E. Guglielmelli, M. J. Johnson, and T. Shibata, "Guest Editorial Special Issue on Rehabilitation Robotics", IEEE Trans. Robot., vol. 25, $\mathrm{n}^{\circ}$. 3, pp. 477-480, $2009 . \quad$ DOI: https://doi.org/10.1109/tro.2009.2022552

6. K. Chen, M. Trkov, J. Yi, Y. Zhang, T. Liu, and D. Song, "A Robotic Bipedal Model for Human Walking with Slips", 2015 IEEE Int. Conf. Robot. Autom., pp. 6301-6306, 2015. DOI: https://doi.org/10.1109/icra.2015.7140084

7. C. Méndez, Y. Aoustin, C. Rengifo, and J. A. Ordoñez, "Evaluation of the Aid Provided by an Exoskeleton in the Reduction of the Joint Torques Exerted by Human Lower Limbs: A Simulation Study", IEEE Lat. Am. Trans., vol. 13, $\mathrm{n}^{\circ}$. 2, pp. 428-433, $2015 . \quad$ DOI: https://doi.org/10.1109/tla.2015.7055560

8. C. L. Vaughan, "Theories of bipedal walking : an odyssey", J. Biomech., vol. 36, pp. 513-523, 2003. DOI: https://doi.org/10.1016/s0021-9290(02)00419-0

9. S. Kajita, M. Morisawa, K. Harada, K. Kaneko, F. Kanehiro, K. Fujiwara, H. Hirukawa, "Biped walking pattern gnerator allowing auxiliary ZMP control", Proc. 2006 IEEE/RSJ Int. Conf. Intell. Robot. Syst., vol. 2, pp. 2993-2999, 2006. DOI: https://doi.org/10.1109/iros.2006.282233

10. C. Chevallereau and Y. Aoustin, "Optimal reference motions for walking of a biped robot", Proc. - IEEE Int. Conf. Robot. Autom., vol. 2005, n. September 2001, pp. 2002-2007, 2005. DOI: https://doi.org/10.1109/robot.2005.1570407

11. T. Sugihara, "Dynamics morphing from regulator to oscillator on bipedal control", Intelligent Robots and Systems, 2009. IROS 2009. IEEE/RSJ International Conference on, pp. 29402945, 2009. DOI: https://doi.org/10.1109/iros.2009.5354582

12. B. Kaddar, Y. Aoustin, and C. Chevallereau, "Contribution de l'effet du balancement des bras sur la marche d'un robot bipède", 2012.

13. A. Hamon, "Influence de la cinématique d'une articulation de genou polycentrique sur la marche d'un robot bipède", Université de Nantes, 2011.

14. S. Alfayad, "Robot humanoïde hydroïd: Actionnement, structure cinématique et stratégie de contrôle", 2009. 
15. E. P. Hanavan, "A mathematical model of the human body," Air force aerospace medical research lab wright-patterson afb oh, 1964.

16. S. Miossec, "Contribution à l'étude de la marche d'un bipède", Ecole Centrale de Nantes (ECN); Université de Nantes, 2004.

17. W. Khalil and E. Dombre, Modeling, identification and control of robots. Butterworth-Heinemann, 2004. DOI:https://doi.org/10.1016/b978-190399666-9/50009-9

18. M. Vukobratović, B. Borovac, and V. Potkonjak, "Towards a unified understanding of basic notions and terms in humanoid robotics", Robotica, vol. 25, $\mathrm{n}^{\circ}$. 1, pp. 87-101, 2007. DOI: https://doi.org/10.1017/s0263574706003031

19. M. H. P. Dekker, Zero-moment point method for stable biped walking. 2009.

20. Y. Aoustin and A. M. Formal'skii, "On optimal swinging of the biped arms," in Intelligent Robots and Systems, 2008. IROS 2008. IEEE/RSJ International Conference, pp. 2922-2927, 2008. DOI:https://doi.org/10.1109/iros.2008.4650725

21. G. Bessonnet, P. Seguin, and P. Sardain, "A parametric optimization approach to walking pattern synthesis", Int. J. Rob. Res., vol. 24, $\mathrm{n}^{\circ}$. 7, pp. 523-536, 2005. DOI: https://doi.org/10.1177/0278364905055377

22. D. Tlalolini, Y. Aoustin, and C. Chevallereau, "Design of a walking cyclic gait with single support phases and impacts for the locomotor system of a thirteen-link $3 d$ biped using the parametric optimization", Multibody Syst. Dyn., vol. 23, n. 1, p. 33, 2010. DOI: https://doi.org/10.1007/s11044-009-91751

23. J. E. Bobrow, F. C. Park, and A. Sideris, "Recent advances on the algorithmic optimization of robot motion", Fast Motions in Biomechanics and Robotics, Springer, pp. 21-41, 2006. DOI: https://doi.org/10.1007/978-3-540-36119-0_2 